\title{
Single-Cell Sequencing of Mitochondrial Mutations Traces Human Cell Lineages
}

\author{
Alison $\mathrm{Liu}^{1}$ \\ ${ }^{1}$ Affiliation not available
}

April 28, 2020

A new method (Ludwig et al., 2019) traces the cellular relationship and hierarchies (the "pedigrees") of human cells within the body by reading the DNA sequences of hundreds to thousands of mitochondria extracted from single cells.

Various genetic labeling techniques have been developed for lineage tracing in other model organisms. However, the above techniques are not applicable in intact humans. Cell lineage tracing is the most direct way to understand the development of complex cell types and their relationships in an organism, and an important method to trace abnormal cells over time to monitor developmental mosaicism, as demonstrated in C. elegans. In mammals, cell lineage tracing is particularly important for tracing cancer cells and their migration because cancers present special difficulties due to fast-paced proliferation and sequential genetic mutations. Lineage tracing can also determine if transplantation is successful and transplanted cells or tissues are on the correct site.

The researchers showed that single-cell RNA sequencing (RNA-seq) and transposase accessible chromatin sequencing (ATAC-seq) methods could be used in combination to trace the inheritance of mitochondrial mutations, chromosomal states, and gene expressions at the same time, in multiple human cell colonies obtained from cultured cells, multiple human tissues, tumor cells, and transplanted cells. ATAC-seq detects the regions of chromosomes that are not wrapped into nucleosomes by histone proteins, thus defining cellular or chromosomal states. Using this method, they identified large numbers of mitochondrial DNA mutations and heteroplasmy (the presence of different types of mitochondrial genomes) that were associated with specific cell populations, tissues, or individuals. These experiments led to an important conclusion that mitochondrial mutations were inherited in the cellular colonies with extensive divisions stably and without being affected by cellular or chromosomal state, and the high mutation rate in mitochondrial DNA allows cellular sub-colonies to be traced with high resolution.

Thus, the single-cell sequencing of mitochondrial DNA mutations provides a method that is much more accurate, stable, and affordable than a single-cell genome sequencing method to study clonal architecture in human health and diseases.

\section{Author ORCiD}

Alison Liu https://orcid.org/0000-0003-0171-6441 


\section{References}

Lineage Tracing in Humans Enabled by Mitochondrial Mutations and Single-Cell Genomics. (2019). Cell, 176 (6), 1325-1339.e22. https://doi.org/10.1016/j.cell.2019.01.022 